I nt $r$ at $r$ acheal admini st $r$ at $i$ on of

thi $r d$-gener at i on I ent i vi rus vect or encodi ng

MPT51 from Mycobact er i um t uber cul osi s i nduces specific CD8+ T-cel I responses in the I ung

\begin{tabular}{|l|l|}
\hline 著者 & Hashi not o Dai \\
\hline $\begin{array}{l}\text { j our nal or } \\
\text { publ i cat i on t i t l e }\end{array}$ & Vacci ne \\
\hline vol une & 26 \\
\hline nunber & 40 \\
\hline page r ange & $5095-5100$ \\
\hline year & 2008-09-19 \\
\hline URL & ht t p: //hdl . handl e. net /10271/829 \\
\hline
\end{tabular}




\section{Intratracheal administration of third-generation lentivirus vector encoding MPT51 from Mycobacterium tuberculosis induces specific CD8+ T-cell responses in the lung}

Dai Hashimoto ${ }^{\mathrm{a}}$, Toshi Nagata ${ }^{\mathrm{b}}$, Masato Uchijima $^{\mathrm{c}}$, Shintaro Seto ${ }^{\mathrm{c}}$, Takafumi Suda $^{\mathrm{a}}$, Kingo Chida ${ }^{\mathrm{a}}$, Hiroyuki Miyoshi ${ }^{\mathrm{d}}$, Hirotoshi Nakamura ${ }^{\mathrm{a}}$, Yukio Koide ${ }^{\mathrm{c}, *}$

${ }^{\mathrm{a}}$ Department of Internal Medicine, ${ }^{\mathrm{b}}$ Department of Health Science, ${ }^{\mathrm{c}}$ Department of Infectious Diseases, Hamamatsu University School of Medicine, 1-20-1 Higashi-ku, Handa-yama, Hamamatsu 431-3192, Japan ${ }^{\mathrm{d}}$ Bio Resouse Center, RIKEN Tsukuba Institute, Tsukuba 305-0074, Japan

*Corresponding author. Tel.: +81 53435 2334; fax: +81 534352335

E-mail address: koidelb@hama-med.ac.jp (Y. Koide) 


\begin{abstract}
The present study evaluates the potential of improved third-generation lentivirus vector with respect to their use as an in vivo-administered T-cell vaccine against tuberculosis. Intratracheal administration of the lentivirus vector encoding MPT51 of Mycobacterium tuberculosis could induce MPT51-specific CD8+ T cells in the mediastinal lymph nodes 2 weeks after the administration. The vaccination could generate MPT51-specific memory CD8+ T cells in the lung, but not in the lymph nodes. Further, a single intratracheal immunization of MPT51 lentiviral vaccine decreased significantly the number of virulent $M$. tuberculosis in the lung after intratracheal challenge of the bacillus. These findings suggest that intratracheal immunization of the third-generation lentiviral vaccines is a promising vaccination strategy against pulmonary tuberculosis.
\end{abstract}

Key words: Intratracheal immunization, Lentivirus, MPT51, Mycobacterium tuberculosis 


\section{Introduction}

Tuberculosis (TB) has been a major cause of death by infectious diseases worldwide. There were an estimated 8.8 million new TB cases in 2005, and 1.6 million people died of TB [1]. An attenuated strain of Mycobacterium bovis Bacillus Calmette-Guérin (BCG) is only currently available anti-TB vaccine which is effective against the severe child forms of TB, yet its efficacy against pulmonary TB in adult is controversial [2]. It is evident that there is an urgent need for a novel and more reliable anti-TB vaccine [3].

Although the mechanisms of protection against TB have not been completely determined, cell-mediated immunity plays an important role in the control of M. tuberculosis infection. There is mounting evidence that type 1 helper T cells are involved in the development of resistance to the disease, primarily through the production of macrophage-activating cytokines, such as interferon- $\gamma($ IFN- $\gamma)$ [4]. In addition, CD8+ cytotoxic T-lymphocytes (CTL) contribute to disease resistance since susceptibility to $M$. tuberculosis is greater in mice deficient in CD8+ T cells [5].

Dendritic cells (DC) are the most potent antigen-presenting cells. DC capture bacteria and other pathogens. Then, they migrate to regional lymphoid organs, where they present antigens (Ag) to naïve T cells [6]. DC are also known to confer T cells the ability to home to non-lymphoid sites. Activated effector/memory T cells migrate preferentially to tissues that are connected to the secondary lymphoid organs where Ag first encountered [7]. In this context, intratracheal vaccination is an attractive option to induce protective immunity against $\mathrm{TB}$ at the lung. In fact, $M$. bovis BCG administered via the respiratory route has been shown to be more effective than when it was given subcutaneously [8-11]. However, intratracheal administration of $M$. bovis BCG may cause severe inflammation in the trachea. For the intratracheal vaccination, such risk of adverse reactions should be avoided. The development of recombinant viral vector systems for gene therapy has prompted examination of their efficacy in gene delivery 
to DC and in direct immunization. Adenovirus vectors were shown to deliver Ag genes to DC. However, pre-existing immunity to viral proteins expressed by the vector prevented effective immunization [12]. Retroviral vectors based on murine leukemia virus have been employed to express Ag in DC [13]. However, the retroviral vectors only infect dividing cells.

Lentiviral vectors have been shown to efficiently transduce a variety of nondividing cells, including DC [14]. Successful transduction of DC with lentiviral vectors has been reported [15-17]. In addition, lentiviral vectors pseudotyped with minimal filovirus envelopes have been reported to increase gene transfer in murine lung [18]. Third-generation self-inactivating (SIN) lentiviral vector was chosen in this study because of its advanced safety profile, allowing its administration in vivo, and because of the presumed absence of pre-existing anti-vector immunity.

Our aim was to develop third-generation lentivirus vectors that express an M. tuberculosis Ag and efficiently induce cell-mediated immunity against pulmonary TB by the intratracheal instillation. As a target Ag, we employed MPT51, the protective character of which we have shown in our previous report [19].

\section{Materials and methods}

\subsection{Mice}

BALB/c mice (8 to14 weeks of age; Japan SLC; Hamamatsu, Japan) were maintained in the Animal Facility of Hamamatsu University School of Medicine. All animal experiments were performed according to the Guidelines for Animal Experimentation, Hamamatsu University School of Medicine.

\subsection{Lentivirus vector production}

The improved third generation lentivirus system had been developed [14, 20, 21]. The system comprised of following plasmids. pCAG-HIVgp is a packaging plasmid in which all accessory genes (vif, vpr, vpu, and nef) and regulatory genes (tat and rev) are deleted. 
pCMV-VSV-G-RSV-Rev is an expression plasmid for vesicular stomatitis virus G glycoprotein and Rev protein. The SIN plasmid, pCSII-CMV-MCS-IRES-EGFP contains a multiple cloning site and the gene encoding enhanced green fluorescent protein (EGFP). MPT51 DNA fragment was inserted into the vector, resulted in pCSII-CMV-MPT51-EGFP. The MPT51 recombinant lentivirus vector was generated by transient transfection of 293T cells with pCAG-HIVgp (10 $\mu \mathrm{g})$, pCMV-VSV-G-RSV-Rev (10 $\mu \mathrm{g})$, and pCSII-CMV-MPT51-EGFP (17 $\mu \mathrm{g})$ plasmids using 10-cm dishes with DoFect-GT1 (Dojindo, Kumamoto, Japan) transfection reagent. $293 \mathrm{~T}$ cells were cultured in Dulbecco's modified Eagle medium (DMEM; Sigma-Aldrich, St. Louis, MO, USA) containing 10\% heat-inactivated fetal calf serum (FCS; Invitrogen, Carlsbad, NM, USA). Culture supernatants were collected every $24 \mathrm{~h}$ for 3 days, filtered through a $0.45-\mathrm{mm}$ pore size filter, and concentrated by two-times ultracentrifugation at $50000 \times \mathrm{g}$ at $20^{\circ} \mathrm{C}$ for $120 \mathrm{~min}$. The viral supernatants were concentrated 1000 times with the ultracentrifugation, finally resuspended in sterile phosphate-buffered saline (PBS), and stored at $-80^{\circ} \mathrm{C}$ until use. The virus titers were determined on 293T cells by measurement of EGFP expression using flow cytometry. Titers of 1 to $2 \times 10^{8}$ infectious units (IU) $\mathrm{ml}^{-1}$ were usually obtained through the experiments.

\subsection{Intratracheal administration}

Mice were anesthetized with an intraperitoneal administration of $0.075 \mathrm{mg}$ ketamine/0.015 mg xylazine per gram weight of mouse. Intratracheal administration of $5 \times 10^{6}$ IU of MPT51 lentivirus in $50 \mu \mathrm{l}$ of sterile PBS was performed by infusion through the vocal cords using a fiber optic light source (LG-PS2, Olympus Optical, Tokyo, Japan) for illuminating the entrance into the trachea [22, 23].

\subsection{Bronchoalveolar lavage (BAL)}

Mice were killed and a midline incision was made to expose the trachea. An 18-G catheter was inserted into the trachea, and the lungs were lavaged with $5 \mathrm{ml}$ of ice-cold sterile PBS. Lavage cells were collected by centrifugation at $300 \times \mathrm{g}$ for $10 \mathrm{~min}$ at $4^{\circ} \mathrm{C}$ and washed with PBS. 


\subsection{Lung tissue lymphocyte isolation}

Lungs were removed from mice, transported in RPMI 1640 medium (5 ml per lung; Sigma-Aldrich), and cut into small pieces $\left(1-2 \mathrm{~mm}^{2}\right)$ with a forceps. Tissue pieces were digested with 3500 dornase units $\mathrm{ml}^{-1}$ of DNase I (Calbiochem, Darmstadt, Germany) and 75 units $\mathrm{ml}^{-1}$ of collagenase type II (Invitrogen) at $37^{\circ} \mathrm{C}$ for $2 \mathrm{~h}$. The digest was filtrated through a $70-\mu \mathrm{m}$ nucleopore filter and centrifuged $(300 \times g, 10 \mathrm{~min})$. The cell pellets were resuspended in PBS containing 0.01 M EDTA and chilled on ice for $5 \mathrm{~min}$, and then subjected to centrifugation in Ficoll-Paque Plus solution (Amersham Pharmacia Biotech, Uppsala, Sweden) at $400 \times g$ and $20^{\circ} \mathrm{C}$ for $30 \mathrm{~min}$. The pulmonary mononuclear cell interface was collected, washed twice, and resuspended in $5 \mathrm{ml}$ of RPMI 1640 medium containing 10\% FCS (RPMI/10FCS) [24]. 2.6. Analysis of CD8+ T cells using MPT51 p24-32 peptide/H2-D ${ }^{d}$ tetramer complex

An MPT51 p24-32 peptide/H2-D ${ }^{\mathrm{d}}$ tetramer complex was kindly supplied by the NIH Tetramer Facility. Cells were treated ammonium chloride and potassium chloride (ACK) lysis buffer for 5 min at room temperature to remove erythrocytes and washed twice with RPMI 1640 medium and resuspended in RPMI/10FCS. The $1 \times 10^{6}$ cells were stained with phycoerythrin (PE)-conjugated MPT51 p24-32 peptide/H2-D ${ }^{\mathrm{d}}$ tetramer complex, fluorescein isothiocyanate (FITC)-conjugated anti-CD8 (53-6.7; BD PharMingen, San Diego, CA, USA), and PE-Cy5-conjugated anti-CD4 (RM4-5; BD PharMingen) monoclonal antibodies (mAb) at $4^{\circ} \mathrm{C}$ for 30 min. After washing, the cells were resuspended in PBS containing $0.1 \%$ sodium azide and $1 \%$ bovine serum albumin, and then analyzed on an EPICS digital flow cytometer (EPICS XL; Beckman Coulter, Miami, FL, USA).

\subsection{Quantification of IFN- $\gamma$ with cytokine enzyme-linked immunosorbent assay (ELISA)}

Spleen cells were harvested from the immunized mice. Recovered cells were plated in 24-well plates at $2 \times 10^{6}$ cells per well in the presence or absence of $1 \mu \mathrm{M}$ of MPT51 p24-32 peptide for 5 days. Concentration of IFN- $\gamma$ in the culture supernatants was determined by a 
sandwich ELISA as described in our previous report [25].

\subsection{Protection assay against M. tuberculosis infection}

Immunized mice were subjected with intratracheal injection of $1 \times 10^{4} \mathrm{CFU}$ of M. tuberculosis H37Rv 10 weeks after MPT51 lentivirus immunization. Mice were sacrificed 5 weeks later and the bacterial numbers in the lung were counted in CFU on Middlebrook 7H10 medium (Becton Dickinson, Sparks, MD, USA). M. tuberculosis H37Rv was kindly donated by Dr. Isamu Sugawara (Research Institute of Tuberculosis, Tokyo, Japan).

\subsection{Statistics}

Data from multiple experiments were expressed as the means \pm SD. Statistical analyses were performed by using StatView-J 5.0 statistics program (SAS Institute, Inc., Cary, NC, USA). Data were analyzed with one factor-analysis of variance followed by the Fisher's protected least significant difference (PLSD) test.

\section{Results}

3.1. EGFP expression of cells in bronchoalveolar lavage fluid (BALF) of mice intratracheally-immunized with MPT51 lentivirus

The lentivirus vector used in this study was pseudotyped with vesicular stomatitis virus glycoprotein and thus was taken up through the normal endocytotic pathway. Therefore, it is able to transduce a wide variety of cells. We first examined EGFP expression of cells in BALF after intratracheal administration of MPT51 lentivirus vector vaccine. As shown in Fig. 1, EGFP expression was observed 1 week after lentivirus administration and the peak of expression was reached around two weeks after the administration. This observation indicates that the cells in BALF, most of which are macrophages, are transduced by the lentiviral vector and that protein expression of transduced vector requires at least 1 week after the administration. We also examined EGFP expression in the mediastinal lymph nodes (MLN). Preferential EGFP 
expression in $\mathrm{CD}_{11 \mathrm{c}^{+}}$cells in the MLN was observed 2 weeks after the administration (data not shown).

\subsection{Induction of MPT51-specific CD8+ T cells in the MLN}

To address whether intratracheal administration of lentiviral vector vaccine results in the induction of MPT51-specific CD8+ T cells in tissues, CD8+ T cells were monitored in the lung, MLN, and the spleen by staining with an MPT51 p24-32 peptide/H2- $\mathrm{D}^{\mathrm{d}}$ tetramer. As shown in Fig. 2, kinetic analysis revealed that the Ag-specific CD8+ T cells appeared 2 weeks after the administration in the MLN and the peak of response was reached around 3 weeks after that. In contrast, there appeared no detectable Ag-specific CD8+ T cells in the lung and the spleen until 6 weeks after the administration.

\subsection{Detection of MPT51-specific memory CD8+ T cells in the lung}

We then examined the lung cells derived from mice intratracheally-immunized with MPT51 lentivirus for detection of MPT51-specific memory CD8+ T cells. We isolated mononuclear cells from lungs of the immunized mice 10 weeks after the administration and stimulated them by MPT51 p24-32 peptide for 5 days. Then, expansion of MPT51-specific CD8+ T cells in the lung and the MLN was evaluated in flow cytometry with MPT51 p24-32/H2-D ${ }^{\mathrm{d}}$ tetramer and anti-CD8 mAb staining. As shown in Fig. 3, expansion of MPT51-specific CD8+ T cells was observed in the lung of MPT51 lentivirus-immunized mice, but not in the MLN of the immunized mice and in naïve mice, indicating that MPT51 lentivirus intratracheal administration was able to induce MPT51-specific CD8+ memory cells in the lung.

\subsection{Induction of protective immunity against M. tuberculosis by intratracheal MPT51 lentivirus} administration

We finally evaluated the effects of intratracheal administration of MPT51 lentiviral vector on induction of protective immunity against $M$. tuberculosis infection. We intratracheally administered $1 \times 10^{4} \mathrm{CFU}$ of $M$. tuberculosis H37Rv to mice 10 weeks after MPT51 lentivirus 
immunization. Five weeks after $M$. tuberculosis challenge, lungs were prepared from the mice and the CFU of M. tuberculosis were evaluated. The CFU in MPT51 lentivirus-immunized mice were significantly lower than those of naïve mice as shown in Fig. 4.

\section{Discussion}

The present study evaluated the potential of third-generation lentivirus vector with respect to the use as mucosal anti-TB T-cell vaccine. From data described above, we were able to draw the following conclusions. (1) The intratracheal administration of the lentivirus vector encoding MPT51 from M. tuberculosis is capable of inducing specific CD8+ T cells in the MLN. MPT51-specific memory CD8+ T cells appear in the lung, but not in the MLN. (3) A single intratracheal immunization of MPT51 lentiviral vaccine decreased significantly the number of virulent $M$. tuberculosis in the lung after intratracheal challenge of the bacillus.

The development of a variety of TB vaccine systems has been reported in order to obtain more effective TB vaccines over BCG vaccine, which is a gold standard of TB vaccine for the time being. Virus-based vaccine strategy is one of them. Vaccinia virus-mediated TB vaccines have been reported. Z Zhu and colleagues [26] showed that immunization with 19kDa- and 38kDaglycol-lipoproteins of $M$. tuberculosis reduced the bacterial numbers of virulent $M$. tuberculosis bacillus in the lungs of immunized mice. Vaccinia virus-based vaccines have been successfully used as a boosting vaccine following BCG- or DNA-based priming vaccination [27]. Adenoviral TB vaccines have been also examined in Dr. Xing’s group [28, 29]. Wang and colleagues [28] reported that a single mucosal, but not parenteral, immunization with recombinant adenoviral-based TB vaccine encoding antigen 85A of $M$. tuberculosis provides potent protection from pulmonary tuberculosis.

We here showed that a single intratracheal MPT51 lentivirus administration was effective for inducing antigen-specific CD8+ T-cell responses in the lung. To our knowledge, it is the first 
report of lentivirus-based vaccine trial for TB. Esslinger and colleagues [30] showed that lentiviral vector injection into the footpad of mice transduced DC that appears in the draining lymph node and in the spleen. They showed that in vivo administration of lentivector was superior to transfer of transduced DC or peptide/adjuvant vaccination in terms of both amplitude and longevity of the CTL response. The results in this present study further showed the effectiveness of lentiviral vector system for mucosal T cell-based vaccination.

Mucosal immunization studies have been reported against airway $M$. tuberculosis infection. Gallichan and Rosenthal [31] showed that long-term mucosal CTL memory was observed only with mucosal, but not systemic, immunization with an adenoviral herpes vaccine. Among a variety of mucosal immunization routes, intranasal vaccination is one of the promising immunization routes for various TB vaccines [9-11, 28, 29]. Giri and colleagues [32] showed that intranasal vaccination with antigen 85A and antigen 85B of M. tuberculosis induced a significantly higher level of IFN- $\gamma$, interleukin (IL)-12, and IL-4 in cervical lymph nodes over subcutaneous vaccination. Further, intranasal vaccination with these Ag imparted protection against $M$. tuberculosis comparable with that obtained from intranasal or subcutaneous $M$. bovis BCG immunization. Another route for eliciting mucosal immunity in the lung is intratracheal administration of vaccines [33]. The method of intratracheal injection used in this study is a simple and rapid method without any invasive procedure [22, 23], compared with conventional intratracheal infection method with tracheal incision step (e.g., [34]). The present study showed the feasibility of the intratracheal intubation method of mucosal immunization for airway infections.

We showed here that MPT51-specific memory CD8+ T cells generated in the lung after a single intratracheal instillation. Kamath and colleagues [35] showed that CFP10-specific and TB10.3/4-specific CD8+ T cells are greatly enriched in the lung compared with other sites of infection such as spleen or lymph nodes. They showed that CD8+ T cells are cytolytic in vivo 
and their cytolytic activity could be detected even as late as 260 days after infection. Servina and Flynn [36] also observed that at 1 week post challenge with $M$.tuberculosis via aerosol, over 30\% of CD8+ and CD4+ T cells in the lungs of immune mice expressed the activation marker CD69 and could be restimulated to produce IFN- $\gamma$, showing a rapid response of CD8+ and CD4+ T cells in the lungs following challenge. These rapidly expanded CD8+ T cells in the lung are derived from lung-resident memory CD8+ T cells because we showed that lung mononuclear cells 10 weeks after MPT51 lentivirus administration responded to MPT51 p24-32 peptide in vitro and produced IFN- $\gamma$. The result indicates that memory CD8+ T cells do exists in the lung. But, some of them may be derived from central memory cells in parabronchial lymph nodes, which are recruited to the lung immediately after $M$. tuberculosis challenge, although we could not detect memory CD8+ T cells in the MLN [37].

Our vaccine was capable of reducing the number of CFU challenged by about $50 \%$, which seems to be not enough in terms of clinical relevance. Since we employed a single administration of the vaccine expressing a single protective antigen, MPT51, booster vaccines and the vaccine expressing several protective antigens could be required for the development of clinically effective vaccine.

As memory T cells present in the lung have been reported to contribute mainly to protection of the host from secondary airway infection [38], the results in the present study suggest that lentivirus TB vaccine represents a promising novel TB vaccine capable of potent mucosal T-cell immune responses.

\section{Acknowledgements}

We thank the NIH Tetramer Facility for providing the MPT51 p24-32/H2-D ${ }^{\mathrm{d}}$ tetramer complex, Dr. Isamu Sugawara (Research Institute of Tuberculosis, Tokyo, Japan) for providing $M$. tuberculosis H37Rv strain. This work was supported by Grants-in-Aid for Scientific Research 
and Centers of Excellence (COE) from the Ministry of Education, Culture, Sports, Science and Technology of Japan; by the United States-Japan Cooperative Medical Science Committee.

\section{References}

[1] WHO Report 2007. Global tuberculosis control: surveillance, planning, financing. Geneva: World Health Organization, 2007.

[2] Sterne JAC, Rodrigues LC, Guedes IN. Does the efficacy of BCG decline with time since vaccination? Int J Tuberc Lung Dis 1998; 2(3):200-7.

[3] Kaufmann SH. Is the development of a new tuberculosis vaccine possible? Nat Med 2000; 6(9):955-60.

[4] Kaufmann SHE. Immunity to intracellular bacteria. In: Paul WE, editor. Fundamental Immunology, Fifth Edition. Philadelphia, Lippincott-Raven Publishers, 2003: 1229-61.

[5] Kaufmann SHE, Flynn JL. CD8 T cells in tuberculosis. In: Cole ST, Eisenach KD, McMurray DN, Jacobs WR Jr, editors. Tuberculosis and the Tubercule Bacillus, Washington DC, ASM Press, 2005: 465-74.

[6] Banchereau J, Steinman RM. Dendritic cells and the control of immunity. Nature 1998; 392:245-52.

[7] Mora JR, Cheng G, Picarella D, Briskin M, Buchanan N, von Andrian UH. Reciprocal and dynamic control of CD8 T cell homing by dendritic cells from skin- and gut-associated lymphoid tissues. J Exp Med 2005; 201(2):303-16.

[8] Barclay WR, Busey WM, Dalgard DW, Good RC, Janicki BW, Kasik JE, et al. Protection of monkeys against airborne tuberculosis by aerosol vaccination with bacillus Calmette-Guerin. Am Rev Respir Dis 1973; 107:351-8.

[9] Falero-Diaz G, Challacombe S, Banerjee D, Douce G, Boyd A, Ivanyi J. Intranasal vaccination of mice against infection with Mycobacterium tuberculosis. Vaccine 2000; 18:3223-9. 
[10] Lyadova IV, Vordermeier HM, Eruslanov EB, Khaidukov SV, Apt AS, Hewinson RG. Intranasal BCG vaccination protects BALB/c mice against virulent Mycobacterium bovis and accelerates production of IFN- $\gamma$ in their lungs. Clin Exp Immunol 2001; 126:274-9.

[11] Chen L, Wang J, Zganiacz A, Xing Z. Single intranasal mucosal Mycobacterium bovis BCG vaccination confers improved protection compared to subcutaneous vaccination against pulmonary tuberculosis. Infect Immun 2004; 72(1):238-46.

[12] Brossart P, Goldrath AW, Butz EA, Martin S, Bevan MJ. Virus-mediated delivery of antigenic epitopes into dendritic cells as a means to induce CTL. J Immunol 1997; 158:3270-6.

[13] Nakamura Y, Suda T, Nagata T, Aoshi T, Uchijima M, Yoshida A, et al. Induction of protective immunity to Listeria monocytogenes with dendritic cells retrovirally transduced with a cytotoxic T lymphocyte epitope minigene. Infect Immun 2003; 71(4):1748-54.

[14] Naldini L, Blömer U, Gallay P, Ory D, Mulligan R, Gage FH, et al. In vivo gene delivery and stable transduction of nondividing cells by a lentiviral vector. Science 1996; 272:263-7.

[15] Dyall J, Latouche J-B, Schnell S, Sadelain M. Lentivirus-transduced human monocyote-derived dendritic cells efficiently stimulate antigen-specific cytotoxic $\mathrm{T}$ lymphocytes, Blood 2001;97(1):114-21.

[16] Rouas R, Uch R, Cleuter Y, Jordier F, Bagnis C, Mannoni P, et al. Lentiviral-mediated gene delivery in human monocyte-derived dendritic cells: Optimized design and procedures for highly efficient transduction compatible with clinical constrains. Cancer Gene Ther 2002; 9:715-24.

[17] Zarei S, Abraham S, Arrighi J-F, Haller O, Calzascia T, Walker PR, et al. Lentiviral transduction of dendritic cells confers protective antiviral immunity in vivo. J Virol 2004; 78(14):7843-5.

[18] Fe Medina M, Kobinger GP, Rux J, Gasmi M, Looney DJ, Bates P, et al. Lentiviral vectors 
pseudotyped with minimal filovirus envelopes increased gene transfer in murine lung. Mol Ther 2003; 8(5):777-89.

[19] Miki K, Nagata T, Tanaka T, Kim YH, Uchijima M, Ohara N, et al. Induction of protective cellular immunity against Mycobacterium tuberculosis by recombinant attenuated self-destructing Listeria monocytogenes strains harboring eukaryotic expression plasmids for antigen 85 complex and MPB/MPT51. Infect Immun 2004; 72(4):2014-21.

[20] Miyoshi H, Blömer U, Takahashi M, Gage FH, Verma IM. Development of a self-inactivating lentivirus vector. J Virol 1998; 72(10):8150-7.

[21] Dull T, Zufferey R, Kelly M, Mandel RJ, Nguyen M, Trono D, et al. A third-generation lentivirus vector with a conditional packaging system. J Virol 1998; 72(11):8463-71.

[22] Brown RH, Walters DM, Greenberg RS, Mitzner W. A method of endotracheal intubation and pulmonary functional assessment for repeated studies in mice. J Appl Physiol 1999; 87(6):2362-5.

[23] Sato J, Schorey J, Ploplis VA, Haalboom E, Krahule L, Castellino FJ. The fibrinolytic system in dissemination and matrix protein deposition during a Mycobacterium infection. Am J Pathol 2003; 163(2):517-31.

[24] Bukreyev A, Belyakov IM, Berozofsky JA, Murphy BR, Collins PL. Granulocyte-macrophage colony-stimulating factor expressed by recombinant respiratory syncytial virus attenuates viral replication and increases the level of pulmonary antigen-presenting cells. J Virol 2001; 75(24):12128-40.

[25] Suzuki M, Aoshi T, Nagata T, Koide Y. Identification of murine H2-D ${ }^{\mathrm{d}}$ - and H2- $\mathrm{A}^{\mathrm{b}}$-restricted T-cell epitopes on a novel protective antigen, MPT51, of Mycobacterium tuberculosis. Infect Immun 2004; 72(7):3829-37.

[26] Zhu X, Venkataprasad N, Ivanyi J, Vordermeier HM. Vaccination with recombinant vaccinia viruses protects mice against Mycobacterium tuberculosis infection. Immunolgy 1997; 92(1):6-9.

[27] McShane H, Pathan AA, Sander CR, Keating SM, Gilbert SC, Huygen K, et al. 
Recombinant modified vaccinia virus Ankara expressing antigen 85A boosts BCG-primed and naturally acquired antimycobacterial immunity in humans. Nat Med 2004; 10(11):1240-4.

[28] Wang J, Thorson L, Stokes RW, Santosuosso M, Huygen K, Zganiacz A, et al. Single, mucosal, but not parenteral, immunization with recombinant adenoviral-based vaccine provides potent protection from pulmonary tuberculosis. J Immunol 2004; 173:6357-65.

[29] Santosuosso M, Zhang X, McCormick S, Wang J, Hitt M, Xing Z. Mechanisms of mucosal and parenteral tuberculosis vaccinations: Adenoviral-based mucosal immunization preferentially elicits sustained accumulation of immune protective CD4 and CD8 T cells within the airway lumen. J Immunol 2005; 174:7986-94.

[30] Esslinger C, Chapatte L, Finke D, Miconnet I, Guillaume P, Lévy F, et al. In vivo administration of a lentiviral vaccine targets DCs and induces efficient CD8 ${ }^{+} \mathrm{T}$ cell responses. J Clin Invest 2003; 111(11):1673-81.

[31] Gallichan WS, Rosenthal KL. Long-lived cytotoxic T lymphocyte memory in mucosal tissues after mucosal but not systemic immunization. J Exp Med 1996; 184:1879-90.

[32] Giri PK, Verma I, Khuller GK. Enhanced immunoprotective potential of Mycobacterium tuberculosis Ag85 complex protein based vaccine against airway Mycobacterium tuberculosis challenge following intranasal administration. FEMS Immunol Med Microbiol 2006; 47:233-41.

[33] Kyd JM, Foxwell AR, Cripps AW. Mucosal immunity in the lung and upper airway. Vaccine 2001; 19:2527-33.

[34] Aguilar D, Infante E, Martin C, Gormley E, Gicquel B, Hernandez Pando R. Immunological reponses and protective immunity against tuberculosis conferred by vaccination of Balb/C mice with the attenuated Mycobacterium tuberculosis (phoP) SO2 strain. Clin Exp Immunol 2006; 147:330-8.

[35] Kamath AB, Woodworth J, Xiong X, Taylor C, Weng Y, Behar SM. Cytolytic CD8 ${ }^{+}$T cells recognizing CFP10 are recruited to the lung after Mycobacterium tuberculosis infection. J 
Exp Med 2004; 200(11):1479-89.

[36] Serbina NY, Flynn JL. CD8 ${ }^{+} \mathrm{T}$ cells participate in the memory immune response to Mycobacterium tuberculosis. Infect Immun 2001; 69(7):4320-8.

[37] Sallusto F, Lenig D, Forster R, Lipp M, Lanzavecchia A. Two subsets of memory T lymphocytes with distinct homing potentials and effector functions. Nature 1999; 401:708-12.

[38] Hogan RJ, Zhong W, Usherwood EJ, Cookenham T, Roberts AD, Woodland DL. Protection from respiratory virus infections can be mediated by antigen-specific CD4 ${ }^{+} \mathrm{T}$ cells that persist in the lungs. J Exp Med 2001; 193(8):981-6. 


\section{Figure legends}

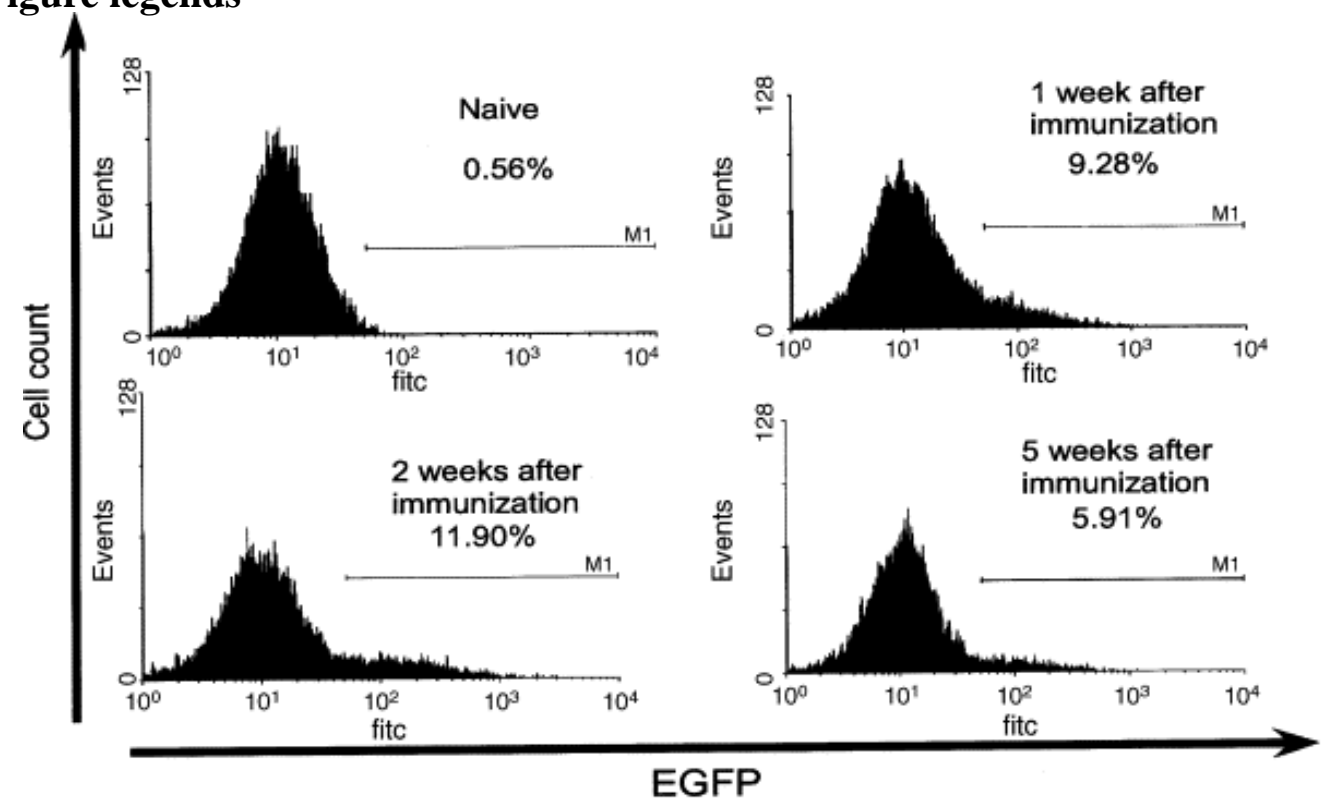

Fig. 1. EGFP expression of cells in BALF of MPT51 lentivirus-administered mice. Mice were intratracheally administered with MPT51 lentivirus and EGFP expression was measured by a flow cytometry every one week for six weeks after MPT51 lentivirus administration. Representative data are shown from three independent data which showed similar results. Percentages in the figure indicate those of EGFP-positive cells in total cells in BALF.

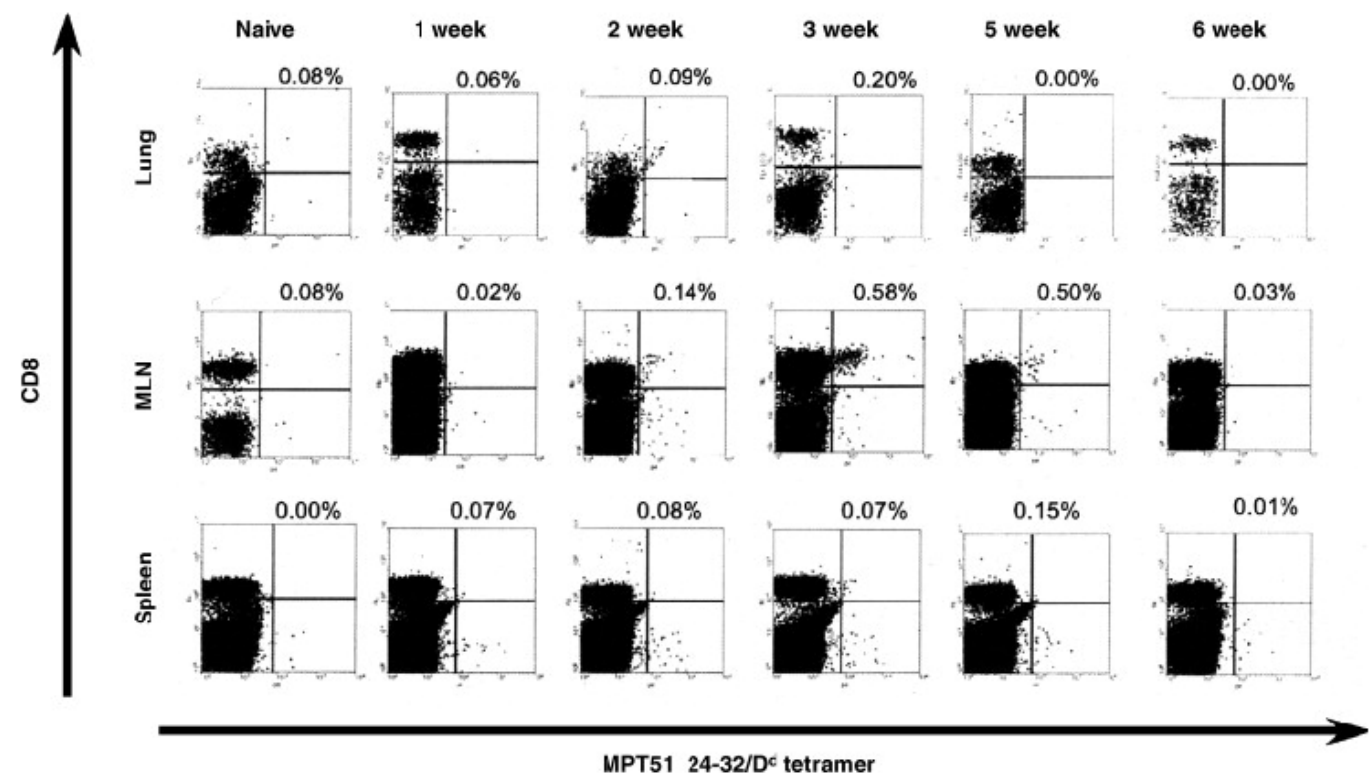

Fig. 2. MPT51 p24-32-specific CD8+ T cells in the lungs, MLN, and spleens of mice intratracheally-administered with MPT51 lentivirus. Mononuclear cells were harvested from the lungs, MLN, and spleens of immunized mice and double-stained with anti-CD8 mAb and MPT51 p24-32/H2-D ${ }^{\mathrm{d}}$ tetramer and measured by a flow cytometry. Representative data of 1 to 6 weeks after MPT51 lentivirus administration are shown. Percentages in the figure indicate those of tetramer-positive cells in CD8+ cells. 


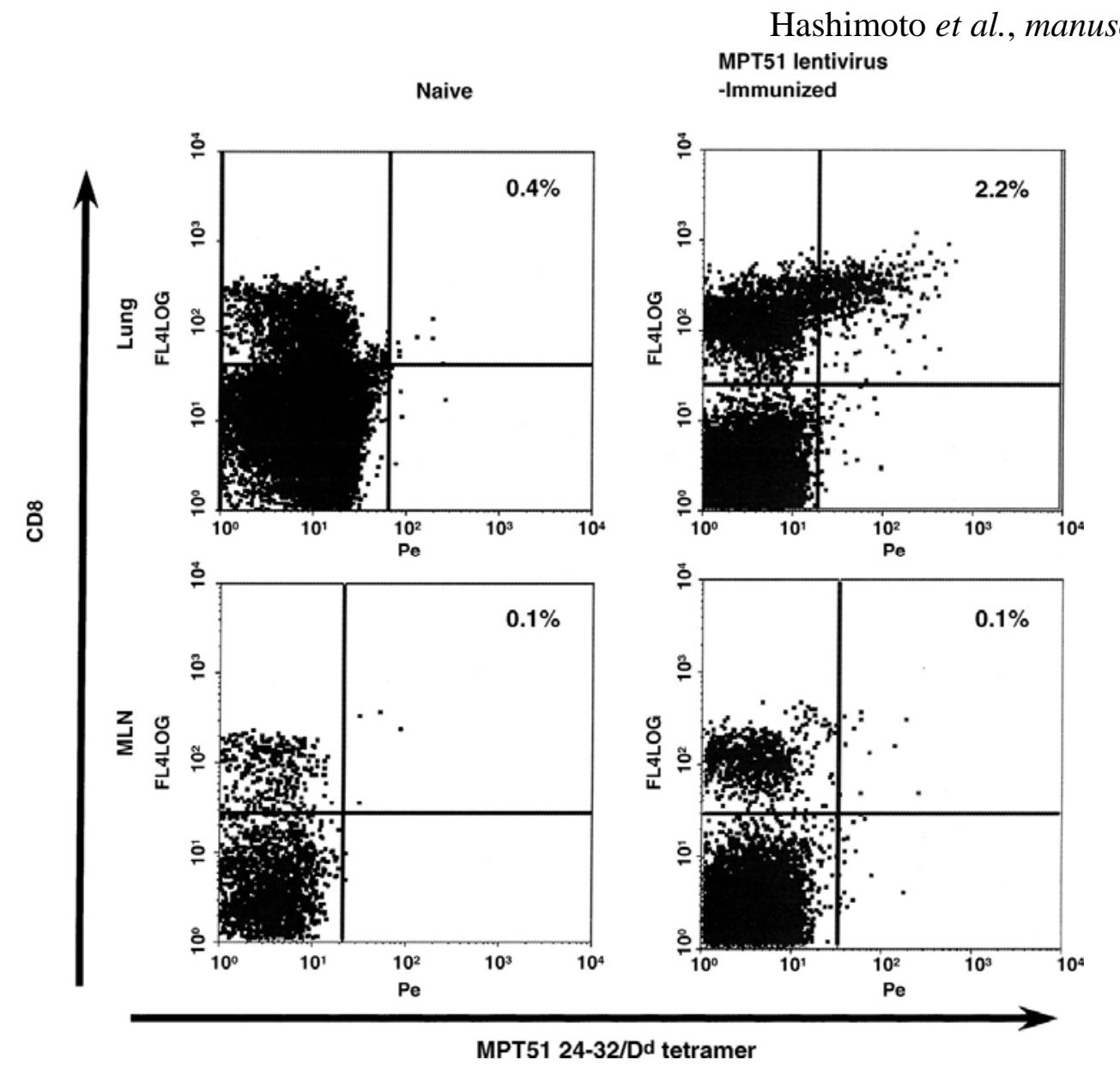

Fig. 3. Detection of MPT51 p24-32-specific memory CD8+ T cells in the lungs of mice intratracheally-administered with MPT51 lentivirus. The lung and the MLN mononuclear cells were harvested from immunized mice 10 weeks after MPT51 lentivirus administration or from naïve mice and double-stained with anti-CD8 mAb and MPT51 p24-32/H2- $\mathrm{D}^{\mathrm{d}}$ tetramer and measured by a flow cytometry. Representative data are shown from three independent data which showed similar results. Percentages in the figure indicate those of the tetramer-positive cells in CD8+ cells.

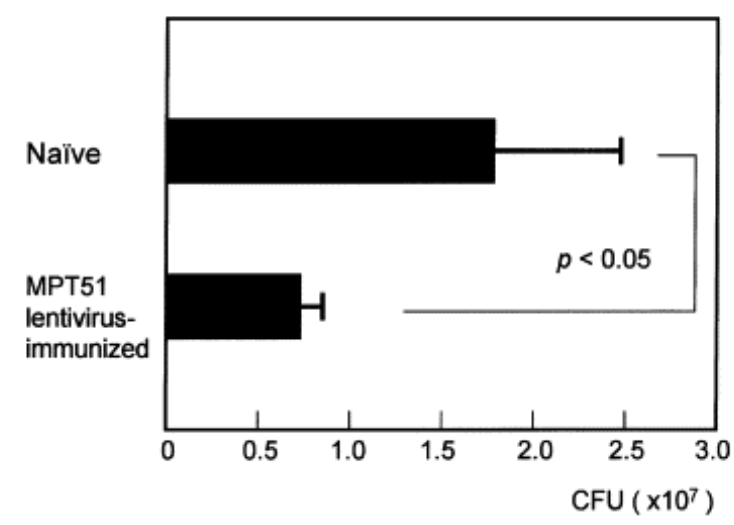

Fig. 4. Induction of protective immunity against virulent $M$. tuberculosis infection with MPT51 lentivirus vaccination. Mice were immunized with a single intratracheal administration of MPT51 lentivirus. The MPT51 lentivirus-immunized mice or naïve mice were challenged with intratracheal injection of $1 \times 10^{4} \mathrm{CFU}$ of $M$. tuberculosis H37Rv. Five weeks after the challenge, the numbers of the challenged bacillus in the lungs were counted. The means \pm SD from 6 mice per each group are shown. 